\title{
A primer of research strategies undertaken in health centers
}

\author{
This article was published in the following Dove Press journal: \\ Journal of Multidisciplinary Healthcare \\ 13 March 2013 \\ Number of times this article has been viewed
}

\author{
Matthew F Hudson \\ Greenville Hospital System, \\ Greenville, SC, USA
}

Correspondence: Matthew F Hudson Greenville Hospital System,

70I Grove Road,

Greenville SC, 29605, USA

$\mathrm{Tel}+\mathrm{I} 8644554845$

Fax + I 8644558404

Email mfhudson@ghs.org
Abstract: This discussion defines various research approaches undertaken in health care or health promotion settings, notably basic science research, translational research, comparative effectiveness research (CER), implementation sciences, and quality improvement. This discussion particularly clarifies the relationship between translational research implementation sciences, comparative effectiveness research (CER), and quality improvement; this discussion further notes how these particular efforts are included in the Health Service Research. The discussion may: (1) introduce novice researchers, practitioners and administrators to various research approaches, (2) establish shared language that enhances comprehension of research strategies sharing similar attributes, and (3) clarify future research direction and resource allocation for health researchers, administrators, and practitioners.

Keywords: basic science, translational research, comparative effectiveness research, implementation sciences, quality improvement, accountable care

\section{Introduction}

Myriad health and wellness stakeholders, clinical practitioners, patients, public health practitioners, insurers, community hospitals, and academic medical centers are pressed with the daunting challenge of optimizing care access and managing costs without compromising quality. This challenge underscores an urgent need for data acquisition and synthesis informing evidence-based medical practice and policy. To facilitate evidence synthesis, stakeholders may initiate strategies varying in rigor, depth, and breadth. For example, practitioners seeking information on a range of issues in a topic may initiate a narrative review of existing literature. ${ }^{1,2}$ Practitioners may also initiate scoping reviews when it is necessary to identify key concepts underpinning a research area, and where it is necessary to clarify the types of evidence available. ${ }^{3,4}$ Systematic reviews are employed when it is necessary to more rigorously discern literature appropriate for review. ${ }^{1}$ This may include a meta-analysis, a process used to systematically merge findings from single, independent studies, using statistical procedures to calculate an overall effect. ${ }^{5}$ Of course, practitioners may also collect data via randomized control trials to examine a clinical question. ${ }^{2}$ Unfortunately, scholarly initiatives are frequently conducted in isolation without duly considering how multiple efforts complement each other. Suboptimal linkages may mitigate more comprehensive problem analyses, data interpretation, and impede care innovation.

Defining research strategies is arguably an antecedent of enhanced connectivity of investigative strategies. This fundamental undertaking may be particularly advantageous given current enthusiasm for investigative strategies such as basic science 
research, translational research, comparative effectiveness research, implementation science, and quality improvement. Therefore, this discussion will explicitly define nascent and perennial investigative strategies, particularly consider where/how these strategies overlap, and posit where these strategies may, collectively, inform approaches to optimizing health care quality access, and cost. This discussion is intended to introduce students, junior researchers, clinicians, and administrators to the research approaches undertaken in health centers. The discussion may standardize vocabulary, thereby enhancing collaboration within and between multidisciplinary care teams. This overview will provide a foundation that may encourage nascent scholars and practitioners to develop a content depth in any or all named research areas. Finally, subsequent scholarship informed by this basic background may enhance information dissemination and scholarly connectivity facilitating evidence-based health care and promotion.

\section{Strategies}

\section{Basic science research}

This type of research includes rigorous inquiry at the molecular level relative to fundamental biology (for example, how a virus is produced, how the virus invades a cell, how the cell responds, etc). Basic science research provides a foundation for disease diagnosis, treatment, and prevention. This work does not focus on social psychological, environmental, or care-delivery issues. Rather, basic science intends to provide insights regarding a disease or condition's biological processes. Therefore, basic science research is frequently considered to occur at the "bench," or laboratory environments. While basic science may adopt a variable role in health service research, Goldstein and Brown ${ }^{6}$ argue medical doctors lacking basic science training are frequently unable to solve biological problems and creatively use new approaches and techniques. Given basic science's imperative to clinical care, it is necessary to sustain scholarship in this area. However, many of health care's currently pressing issues are precipitated less by ignorance about biological processes. Rather, health care needs to become more intentional about translating insights produced via basic science into clinical practice.

\section{Translational research}

Knowledge transfer is a fundamental concern for those seeking to apply basic science insights to clinical care. Translational advocates 7,8 identify two "translational blocks" to the basic science/clinical care interface, thus more explicitly defining translational research. The first translational block involves facilitating the "transfer of new understandings of disease mechanisms gained in the laboratory into the development of new methods for diagnosis, therapy, and prevention and their first testing in humans" (T1). The second translational block is defined as facilitating "the translation of results from clinical studies into everyday clinical practice and health decision making"7 (T2). These two approaches are collectively called translational research. Thus, the T1 component of translational research may clarify the efficacy of a drug or intervention (whether an intervention produces the desired outcome under optimal conditions). ${ }^{9}$ Efficacy insights may be gleaned via randomized clinical trials. The T2 component of translational research arguably addresses the effectiveness of a drug or intervention (whether an intervention works under regular, "real world" circumstances). ${ }^{9}$ Previous work indicates there are considerable delays translating basic science insights into clinical research and practice, regardless of the type of original study, technological promise, and therapeutic and preventive implication. ${ }^{10}$ Consequently, providers, such as academic health centers, may enlist academic and business partners to expedite research innovations into clinical practice. All interventions - drugs or therapies - developed via translational research may be compared to assess which therapy works better under particular conditions; comparative effectiveness research addresses this charge.

\section{Comparative effectiveness research}

The Institute of Medicine defines comparative effectiveness research (CER) as "the generation and synthesis of evidence that compares the benefits and harms of alternative methods to prevent, diagnose, treat, and monitor a clinical condition, or to improve the delivery of care."11 Arguably then, CER compares multiple condition-specific strategies, validated via translational research, on the basis of cost, quality, and access. Assuming CER is predicated upon representative population samples, CER may also clarify whether a given strategy is generally preferred in the population, or whether preferences vary by socio-demographic factors, such as race or ethnicity. Further, CER may also clarify condition of cost/benefit equity; that is, CER may affirm that patient (or system) cost is concomitant with patient (or system) rated quality. Rich ${ }^{12}$ suggests that both the CER acronym, and the ancillary benefit of cost alignment, led some to misinterpret CER's purpose as a cost effectiveness or cost management initiative; many erroneously assumed that fiscal concerns singularly motivated scholarship, thereby minimizing, or at worst, undermining the import of patient preference in 
clinical care decision making. Consequently, CER advocates often frame CER-based scholarship as patient centered outcomes research (PCOR). ${ }^{13}$ PCOR explicitly considers: assessment of preventive, diagnostic, treatment strategies, health care system improvement, communication and dissemination, health care disparities, and methodological innovations. Therefore, CER and PCOR consider the most appropriate "bedside ready" intervention, per patient values and preference. Optimal cost, quality and access assessment (informed by patient preference and care system resources) ultimately determines intervention propriety.

\section{Implementation sciences research}

Eccles et $\mathrm{al}^{14}$ define implementation research as "the scientific study of methods to promote the systematic uptake of clinical research findings and other evidence based practices into routine practice, and hence [sic] to improve the quality (effectiveness, reliability, safety, appropriateness, equity, efficiency) of health care. It includes the study of influences on the healthcare professional and organizational behavior". Thus, where CER determines "which" strategies are advantageous, implementation research determines "how" to best implement the optimal strategies per CER. Presumably, this may include comparing integration processes in the manner CER similarly compares the interventions.

Implementation research is arguably critical to optimal intervention integration. Eccles et $\mathrm{al}^{14}$ acknowledge one challenge to optimal integration is that health care professionals may differ in their perceived need for, and attitudes toward, quality enhancement initiatives. Consequently, evidencebased initiative implementation may vary due to disparate perceptions and attitudes. Ohmann and Kuchinke ${ }^{15}$ also argue clinical research is challenged by inadequate information technology (IT) infrastructure inhibiting information exchange and care implementation. Hence, electronically networking health records and clinical research is an evolving interest area. Others have considered the favorable impact of enhanced IT and interoperability on care continuity and patient safety. ${ }^{16,17}$ Ohmann and Kuchinke similarly posit enhanced connectivity may enhance the scientific competence of multiple research centers, via expedited innovation and reduced research redundancy (horizontal integration); enhanced connectivity may also invigorate research/clinician communication and information exchange immediately applicable to the clinical enterprise (vertical integration). ${ }^{15}$ The focus of implementation research methods and processes may subsequently inform how to align care team beliefs and optimally disseminate evidence-based care triumphs.

\section{Quality improvement}

The Agency for Healthcare Research and Quality (AHRQ) previously defined quality as simply "doing the right thing, at the right time, in the right way, for the right person - and having the best possible results." 18 In instances where one witnesses or assumes suboptimal outcomes, Varkey et $\mathrm{al}^{19}$ note quality improvement (QI) mandates identifying and measuring the undesired outcomes. Subsequent improvement efforts should assess whether initiatives facilitate desired change, contribute to unintended results elsewhere in the system, and require additional efforts to correct undesired process. Varkey et al identify common quality improvement (QI) strategies, and particularly outline a "trial-and-learning" quality improvement strategy, Plan-Do-Study-Act (PDSA). ${ }^{19}$ This includes stating objectives and improvement implementation plan (Plan); initiating the improvement strategy; documenting problems, unexpected variations, and analyzing data (Do); summarizing results (Study); and determining the appropriate changes per results (Act). This process is intended to occur in a rapid cycle designed to produce iterative improvements in process, eventually integrated into standard practice. While this description may clarify "how" to improve quality, the description fails to clarify the climate amenable to initiating such an initiative. Swensen et $\mathrm{al}^{20}$ propose four principles and key components of health system change: optimized culture of safety (standardized patient service predicated upon "best practice" and medical evidence), infrastructure (eg, care team instruction in QI principles, electronic record interface facilitating information dissemination), human factors and systems engineering (a perspective that emphasizes the role of norms, policy, technology, and setting as care outcome mediators), and execution (having teams accountable for initiating improvement initiatives). Health care systems initiating QI in this context may mitigate unwarranted care variation and reduce medical error. More recent work emphasizes the importance of alignment (prioritizing clinical processes to be improved, thereby ensuring adequate resource allocation) and managed diffusion (replication and dissemination of standardized practice). ${ }^{21} \mathrm{QI}$ is typically a health care system mandate, and arguably examines whether optimal strategies (per CER) are appropriately integrated (per implementation research) reliably/consistently.

\section{Accountable care}

The Medicare Payment Advisory Commission ${ }^{22}$ defines accountable care organizations (ACO) as organizations of providers agreeing to accept risk for the cost and quality of care they provide their patients, with their payments adjusted 
through a system of bonuses and penalties applied depending on whether the ACO achieved or failed to meet specified performance. While national metrics are currently subject to debate, bonuses and penalties may be dictated by the evidence supporting care value, as indicated by treatment cost, quality and access propriety. All research enterprises proposed above and in Figure 1 will inform and validate accountable care. Notably, IOM ${ }^{11}$ prioritizes research "Compar[ing] the effectiveness of accountable care systems and usual care on costs, processes of care, and outcomes for geographically defined populations of patients with one or more chronic disease." Consequently, accountable care may warrant its own research strategies, analytic approaches, and economic models to validate the merits of this risk sharing model.

\section{Proposed research model/process}

Figure 1 outlines a continuum of scholarly inquiry that may be conducted in a health care or promotion setting (eg, community hospital, academic health center). The figure depicts a line representing a research continuum targeting three areas: laboratory initiated work (the bench), the point of clinical practice (the bedside), and prescribed practices mediating cost, hospital quality, and patient outcomes (system policy).
Dotted curved arrows at each end of the continuum presume a reciprocal process whereby advances at one end of the continuum facilitate the other-policy may beget bench advances, policy may inform agenda for basic science research.

The figure further specifies five different types of research or scholarly inquiry: basic sciences, translational sciences, comparative effectiveness research, implementation sciences, and quality improvement. Collectively, our research enterprise simply: (1) clarifies fundamentally normal and abnormal human function (basic sciences), (2) posits an appropriate intervention to improve health (translational research), (3) clarifies the preferred intervention among alternatives (comparative effectiveness) per patient preference and values (patient-centered outcomes research), (4) identifies the best means by which to integrate the preferred intervention (implementation sciences), and (5) evaluate care delivery processes to ensure the preferred intervention meets or exceeds outcome expectations; insights collectively facilitate optimal patient care and subsequent patient wellness. Ultimately, these research strategies, independently and collectively, provide insights relative to cost, quality and access. While each research area has an epistemological primacy target, we frame our research enterprise as a continuum, not siloed

\section{Basic science} research-rigorous inquiry at the cellular or molecular level

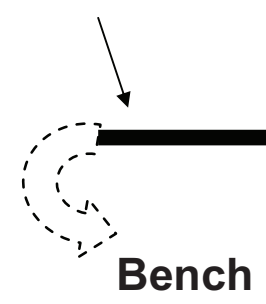
clinical effectiveness scholarship assessing propriety of interventions per patient's values and preferences

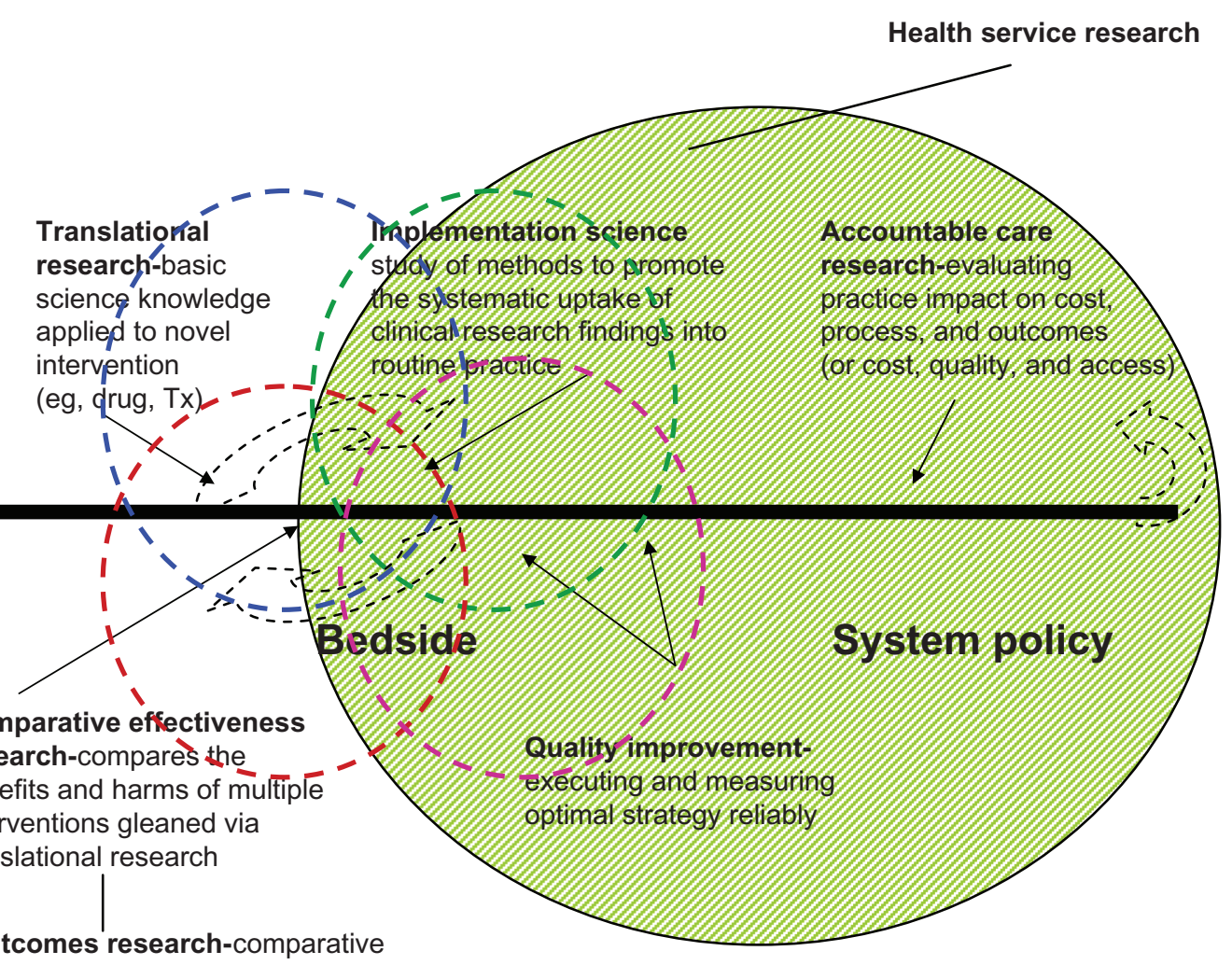

Figure I Scholarly inquiry informing practice and policy. 
scholarly endeavors; each research area is arguably dependent on the other, providing insights derived from, or impacting, bench, bedside, or system policy. Thus, the illustration is not intended to advocate the preeminence of one research agenda over the other.

Figure 1 also depicts dotted circles overlapping the domains of translational research, CER, implementation science and quality improvement. This illustration intends to simulate the conceptual overlap between what care innovators may do to improve health (translational research) and what is best to do (CER). Likewise, there is arguably conceptual overlap between identifying the best way to implement the best procedure (implementation sciences) and considering how to improve the strategy (quality improvement). The dotted circles also intend to simulate a methodological overlap among these four research strategies. Data collection approaches, such as survey, case study, focus group, and chart review, are not exclusive to any of these general research strategies. Consequently, data collection methods used in one research strategy may simultaneously, and subsequently, inform another. Ultimately, these research strategies, independently and collectively, may provide insights relative to cost, quality and access.

\section{How do these research initiatives fit in the domain of health service research?}

The Department of Veterans Affairs (VA) defines health service research as "examining the organization, delivery, and financing of health care from the perspectives of patients, caregivers, providers, and managers to improve the quality and economy of care". ${ }^{23}$ Arguably then, many research primacy targets are included in health service research. While basic science research and translational research informs our system of "what" care our system should provide, health service research (particularly implementation sciences, quality improvement, and accountable care research) informs our system of "how" we may deliver care. Figure 1 models comparative effectiveness research as the interface between health service research and basic science and implementation sciences. Further, CER strategies may overlap with implementation sciences, as CER may compare drugs and devices, as well as strategies designed to improve care delivery.

Each research strategy uniquely contributes to the science and practice of health promotion and disease prevention. However, hospitals and academic health centers may particularly augment efforts and resources relative to health service research. National mandates relative to accountable care, coupled with persisting care disparities and regional care variations, mandate HSR prominence as a system priority and academic priority.

\section{Discussion limitations}

First, the research descriptions and accompanying model are restricted to generic research clarifications per inquiry goal/intent (eg, translation, comparative effectiveness, implementation). The description and model provided do not explicitly consider research strategies researchers may undertake to address their specific aims (eg, case study, survey, focus group, chart review). These strategies are arguably ubiquitous and amenable to any given research domain described above. However, each strategy's strengths and limitations vary in import, per the specific aim addressed. Thus, seminal research texts ${ }^{24-27}$ are better positioned to guide practitioners toward study designs and data collection methods optimally informing their specific aims; this discussion is limited to facilitating awareness of the research context in which care teams initiate care assessment and improvement.

Second, our model and accompanying discussion frames research as a continuum, and suggests health-related scholarship reflects an ordinal progression. Such modeling may minimize the unique impact each research perspective may exert on care innovation. Further, the model's ordinal and linear nature may minimize the possibility that seemingly disparate research perspectives may more directly inform other continuum domains (eg, translational research may directly impact accountable care; comparative effectiveness research may inform basic science research). Indeed, translational research advocates, for example, argue clinical practice should more explicitly inform basic science research. ${ }^{28,29}$ Arguably, this model and the accompanying discussion oversimplifies the nature and impact of scholarly inquiry; the model arguably depicts scholarship and discovery as a linear process. In fact, discovery may occur using any order of the domains discussed. Limitation notwithstanding, this model and discussion may provide a reasonable basis from which a multidisciplinary team may develop a shared vocabulary, perspective, and process facilitating a more tailored approach to scholarly inquiry.

\section{Conclusion}

The discussion above defines research strategies many health care organizations currently undertake. The discussion particularly clarifies the interface between translational research, CER and implementation sciences, and quality improvement. The discussion also asserts health service research is a principal research enterprise in many health care settings, 
but notes basic science's importance and system relevance. Consequently, it behooves health care systems to sustain efforts relative to basic science. A comprehensive approach to scholarly inquiry may elucidate the tenability of accountable care, and enhance care quality. This discussion may help nascent health and wellness promoters align amenable investigative perspectives and strategies to knowledge and improvement aims. This discussion may also assist experts in any single approach to consider adjunct strategies facilitating more robust investigative strategies.

\section{Disclosure}

The author reports no conflicts of interest in this work.

\section{References}

1. Collins JA, Fauser BCJM. Balancing the strengths of systematic and narrative reviews. Hum Reprod Update. 2005;2:103-104.

2. Guyatt G, Drummond R, Meade MO, Cook DJ. JAMA's Users' Guides to the Medical Literature: A Manual for Evidence-based Clinical Practice, 2nd ed. New York; McGraw-Hill Medical; McGraw-Hill; 2008.

3. Arksey H, O’Malley L. Scoping studies: towards a methodological framework. Int Soc Res Methodol. 2005;8(1):19-32.

4. Brien SE, Lorenzetti DL, Lewis S, Kennedy J, Ghali WA. Overview of a formal scoping review on health system report cards. Implement Sci. 2010;5:2-2.

5. Shorten A, Shorten B. What is meta-analysis? Evid Based Nurs. 2013;16(1):3-4.

6. Goldstein JL, Brown MS. The clinical investigator: bewitched, bothered, and bewildered - but still beloved. J Clin Invest. 1997;99(12): 2803-2812.

7. Sung NS, Crowley WF Jr, Genel M, Salber P, et al. Central challenges facing the national clinical research enterprise. JAMA. 2003;289(10):1278-1287.

8. Woolf SH. The meaning of translational research and why it matters. JAMA. 2008;299(2):211-213.

9. Compher C. Efficacy vs effectiveness. JPEN J Parenter Enteral Nutr. 2010;34(6):598-599.

10. Contopoulos-Ioannidis DG, Ntzani E, Ioannidis JPA. Translation of highly promising basic science research into clinical applications. $\mathrm{Am}$ J Med. 2003;114(6):477-484.

11. Institute of Medicine. Committee on Comparative Effectiveness Research. Initial National Priorities for Comparative Effectiveness Research. Washington, DC: Institute of Medicine of the National Academies; 2009.
12. Rich EC. From methods to policy: past as prologue: how comparative effectiveness research became patient-centered outcomes research. Journal of Comparative Effectiveness Research. 2012;1(6):475-477.

13. Selby JV, Beal AC, Frank L. The Patient-Centered Outcomes Research Institute (PCORI) national priorities for research and initial research agenda. JAMA. 2012;307(15):1583-1584.

14. Eccles MP, Armstrong D, Baker R, et al. An implementation research agenda. Implement Sci. 2009;4:18.

15. Ohmann C, Kuchinke W. Future developments of medical informatics from the viewpoint of networked clinical research. Interoperability and integration. Methods Inf Med. 2009;48(1):45-54.

16. Brailer DJ. Interoperability: the key to the future health care system. Health Aff (Millwood). Jan 2005;Suppl:W5-W19.

17. Leape LL, Berwick DM. Five years after To Err Is Human: what have we learned? JAMA. 2005;293(19):2384-2390.

18. US Department of Health and Human Services. Agency for Healthcare Research and Quality. A quick look at quality [webpage on the internet]. http://archive.ahrq.gov/consumer/qnt/qntqlook.htm. Accessed March 21, 2011.

19. Varkey P, Reller MK, Resar RK. Basics of Quality Improvement in Health Care. Mayo Clin Proc. 2007;82(6):735-739.

20. Swensen SJ, Dilling JA, Milliner DS, et al. Quality: the Mayo Clinic approach. Am J Med Qual. 2009;24(5):428-440.

21. Swensen SJ, Dilling JA, Harper CM, Noseworthy JH. The Mayo Clinic value creation system. Am J Med Qual. 2012;27(1):58-65.

22. US Department of Health and Human Services. Medicare Payment Advisory Commission. Medicare Payment Advisory Commission Report to the Congress, Mar 2010 [webpage on the internet]. http://www.medpac. gov/documents/Mar10_EntireReport.pdf. Accessed March 21, 2011.

23. US Department of Veterans Affairs. What is Health Service Research? [webpage on the internet]. http://www.hsrd.research.va.gov/funding/ what-is-hsr.cfm. Accessed March 21, 2011.

24. Campbell DT, Stanley JC, Gage NL. Experimental and Quasi-experimental Designs for Research. Boston, MA, USA: Houghton Mifflin; 1963.

25. Babbie ER. The Practice of Social Research. Belmont, CA, USA: Wadsworth Pub Co, 1993.

26. Fowler FJ. Survey Research Methods. Newbury Park, CA, USA: Sage Publications; 1993.

27. Yin RK. Case study research: design and methods. Thousand Oaks, CA, USA. Sage Publications; 2003.

28. Marincola F. Translational Medicine: A two-way road. J Transl Med. 2003;1(1):1.

29. Nussenblatt RB, Marincola F, Schechter AN. Translational Medicine - doing it backwards. J Transl Med. 2010:8-12.
Journal of Multidisciplinary Healthcare

\section{Publish your work in this journal}

The Journal of Multidisciplinary Healthcare is an international, peerreviewed open-access journal that aims to represent and publish research in healthcare areas delivered by practitioners of different disciplines. This includes studies and reviews conducted by multidisciplinary teams as well as research which evaluates the results or conduct of such teams or

\section{Dovepress}

healthcare processes in general. The journal covers a wide range of areas and welcomes submission from practitioners at all levels, from all over the world. The manuscript management system is completely online and includes a very quick and fair peer-review system. Visit http://www.dovepress.com/testimonials.php to read real quotes from published authors. 